\title{
Structural Mechanical Behavior Analysis Research on Stress/Strain State Study of Vibratory Bowl in Assembly Engineering
}

\author{
Ö. KARAÇALI* \\ Istanbul University Cerrahpaşa, Faculty of Engineering, Department of Mechanical Engineering, \\ Avcrlar, Istanbul, Turkey
}

\begin{abstract}
In modern manufacturing, mechanical assembly in high volume production relies upon the removal of specially positioned components in a line from the vibrating spiral feeder bowl, which feeds the parts and reassembles hundreds of pieces. A bowl with a helical track vibrated by the electromagnetic leaf spring and gravity is employed to move or carry the parts on the non-linear dynamic vibrating spiral feeder bowl. The vibrating spiral feeder bowl depends on vibration correspondingly involving in chaotic regions and frequent repairs. Some of the inadequacies to the vibrating spiral feeder bowl are bowl top surface wear due to friction as a result of repeated impacts, the dynamic behavior for continuous vibration and material structural deformations that affects its accurate performances. To overcome these problems, a model of the located on the electromagnetic leaf spring system was investigated to resolve bowl material, geometric parameters and mechanical stresses under vibrating forces and friction. There is not enough literature available regarding physical parameters related to static stress modeling and von Mises criteria about vibrating bowl and leaf spring. Therefore, the research goal was to study structural static stresses, strain and von Mises failure mode caused by centrifugal stresses, strain and vibrations in ANSYS. The main distinction of this work from previous researches is the analytical analysis of the physical parameters associated to structural and mechanical behavior model of vibrating spiral feeder bowl according to ISO 10816 standards. The cast aliminium material integrated with spring leaf behaviour simulation for the vibrating spiral feeder bowl was analysed by finite element structural static, and von Mises analysis in ANSYS and the computational model was refined analytically.
\end{abstract}

DOI: 10.12693/APhysPolA.135.928

PACS/topics: vibratory spiral bowl feeder, von Mises stresses, cast aluminum, finite element analysis

\section{Introduction}

Moving parts for automatic assembly on the vibrating bowl are among the most problematic engineering tasks and are limited to the operation of the system [1]. Studies related to the vibrating bowl, material behavior of the spiral bowl, geometrical analysis and physical parameters are observed through computational analysis [2]. Loy and Reinhart [3] presented the design, operation, and simulation of a mobile parts feeder. Jiang [4] presented a dynamic model for the linear vibratory feeder and investigated the operating parameters that could affect the conveying velocity of a part. Each spring deformation is complicated as it relies on the possible plastic deformation and torsion alongside the axial and crosswise directions [5]. It is assumed that the leaf spring is in an apparent possible bending when considering the deformation in the thickness direction only [6]. The finite element $(\mathrm{FE})$ analysis and experimental analysis were performed in feeder. In Fig. 1, vibrating spiral feeder bowl (VSFB) leaf spring under research was given for meshed bowl and leaf spring.

*e-mail: ozdogank@istanbul.edu.tr

\section{Method and experimental procedure}

In this research, a standard closed track with suitable width and pitch was used for industrial assembly purposes. The model of the VSFB located above the electromagnetic leaf spring as an integrated system was investigated to determine bowl material, geometric

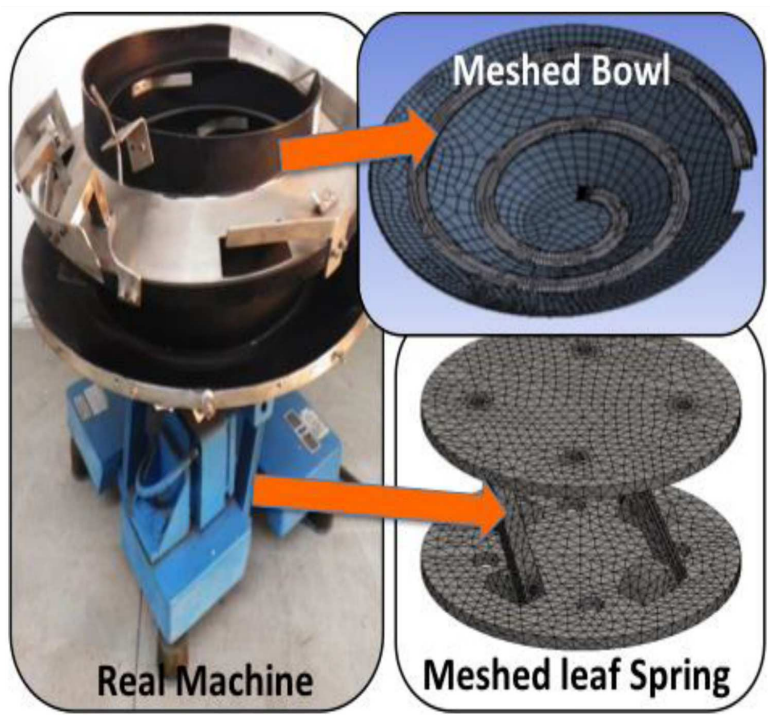

Fig. 1. Vibratory feeder bowel-leaf spring. 
parameters and mechanical stresses under vibrating forces and friction. The goal of this work was to research physical strength, deformability, failure mode and total displacement of the VSFB as displayed partially in Fig. 2. The research was divided into three parts to (1) structural static stresses, (2) strain and (3) von Mises failure mode caused by vibrations and friction and handled according to ISO 10816 standards. In the first step, the finite element $(\mathrm{FE})$ technique is used to measure displacements on the surface of bowl caused by the controlled excitation. In the second step, the bowl size is determined by developing the FEM model of the bowl that produces the same displacements as measured experimentally. A vertical and horizontal displacement of the bowl occurred when solenoid coil energized with rotation movement against the spring system as shown in Fig. 2. The resultant direction caused as $13.48 \mathrm{~mm}$ displacement normal to leaf springs inclination as shown in Fig. 3. The Horizontal displacement strength increases while the center of the bowl circumferentially rotate. To achieve optimization of the geometric parameters of the bowl feeder structure, an increase in feed-rate caused smoother, quieter running; and lower power consumption. ANSYS-finite element simulation models were developed from the SolidWoks drawings as given in Fig. 2.

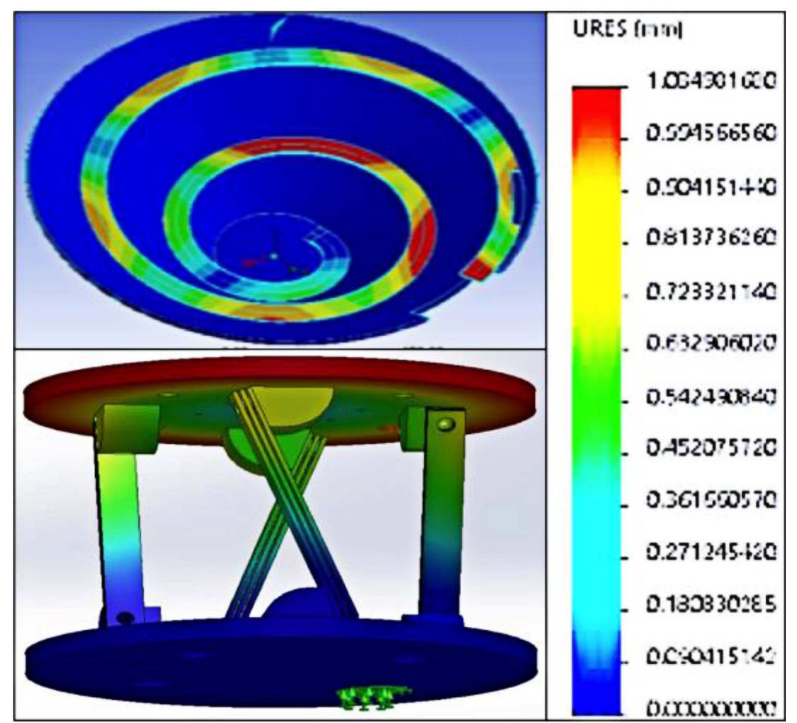

Fig. 2. Total Displacement of VSFB.

The research on the vibrating bowl took considerations into account of the material properties, dimensions, meshing, element type, boundary conditions to deliver total displacement, Strain energy density as shown in Fig. 4. The boundary and loading conditions are: outer edge of the larger circular plate (bottom plate) is constrained completely, the smaller circular plate (top plate) is to be pulled away, and the total applied load is $100 \mathrm{~N}$ applied at the outer edge of the top plate as displayed strain energy criterion simulation in Fig. 4.

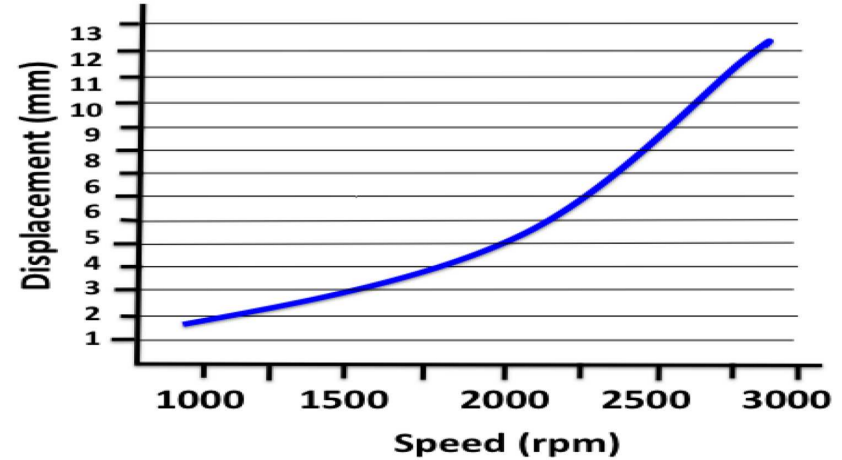

Fig. 3. Speed vs displacement effect of spring.

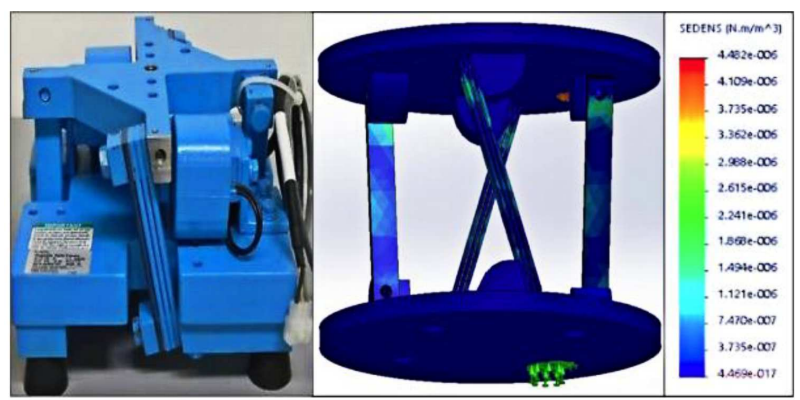

Fig. 4. Leaf spring strain energy.

Investigation into the vibrations and computation of the stress fields is difficult by analytical methods. Recourse to FEM can help in arriving at the values. FEM simulation environment produces the vibratory bowl model and leaf spring driven by electromagnetic system for predicting deflections $(\varepsilon)$, stresses $\left(\sigma_{e}\right)$ and vibrations in the structure. Structural material energy stress $U_{\varepsilon}[7]$ in the direction of $x, y, z$ axis formulated as follows in Eqs. (1)-(5):

$$
\sigma_{e}=\sqrt{\frac{\varepsilon_{1}+\varepsilon_{2}}{3}}
$$

where

$$
\varepsilon_{1}=0.5\left[\left(\varepsilon_{x}-\varepsilon^{*}\right)^{2}+\left(\varepsilon_{y}-\varepsilon^{*}\right)^{2}+\left(\varepsilon_{z}-\varepsilon^{*}\right)^{2}\right]
$$
and

$$
\begin{aligned}
\varepsilon_{2} & =\frac{\left(\varepsilon_{x y}\right)^{2}+\left(\varepsilon_{x z}\right)^{2}+\left(\varepsilon_{y z}\right)^{2}}{4}, \\
\varepsilon^{*} & =\frac{\varepsilon_{x}+\varepsilon_{y}+\varepsilon_{z}}{3}, \\
U_{\varepsilon} & =\sum_{i=1}^{N}\left(\sigma_{x} \varepsilon_{x}+\sigma_{y} \varepsilon_{y}+\sigma_{z} \varepsilon_{z}+T_{x y} \varepsilon_{x y}+T_{x z} \varepsilon_{x z}\right. \\
& \left.+T_{y z} \varepsilon_{y z}\right) \operatorname{Vol}(i) \frac{W(i)}{2} .
\end{aligned}
$$

Structural analysis in this research explored maximum stress $\sigma_{e}$, deflections $\varepsilon$ and material energy strain $U_{\varepsilon}$ in VSFB system. The feasibility of design of VSFB is calculated based on structural design requirements of $\sigma_{e}, \varepsilon$, $U_{\varepsilon}$. By the ANSYS, the von Mises stress and the mesh of 
resultant displacement in case of $2000 \mathrm{rpm}$ were plotted. In order to validate ANSYS tool, the outer part of the bucket of centrifuge structure is considered as the thick cylinder and circumferential stress is calculated. The particular criteria for selected collapsing of the system was the ultimate strain energy criterion and von Mises greatest failure principal. The von Mises stress indicates the realistic data that the material of VSFB will decline when the equivalent stress is exceeded by yield point level. In this research, it was demonstrated that how the FE method can be used to display dynamic behavior in the real model of VSFB, as shown in Fig. 5.

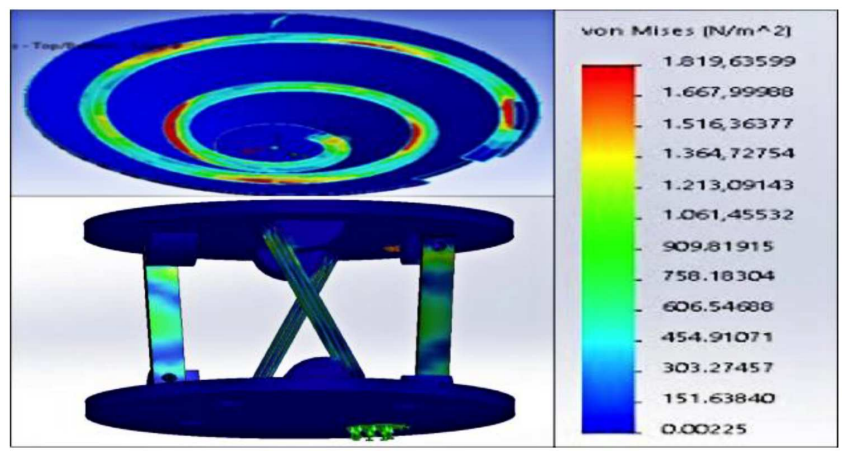

Fig. 5. The von Mises stress analysis of VSFB.

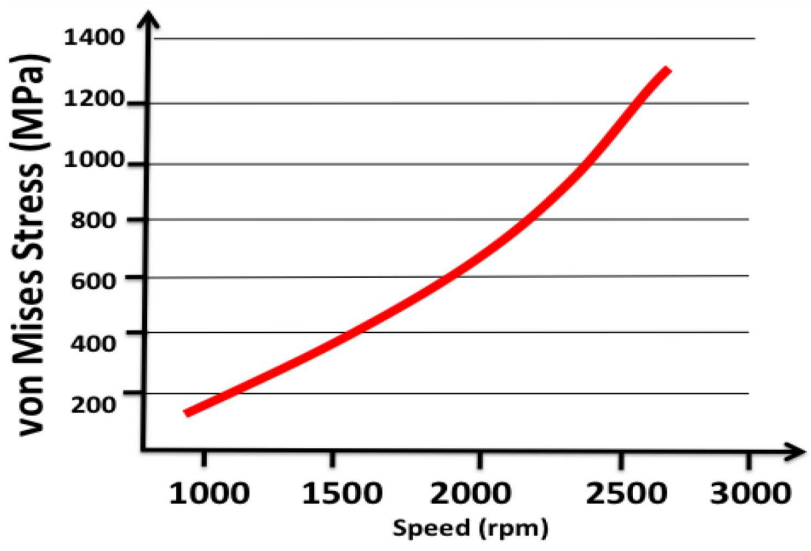

Fig. 6. Speed vs von Mises stress in VSFB.

The feed rate of the VSFB was drastically influenced when the spring has physically failed due to fatigue or unknown means of damage. A crack may not always be visible to the eye but FEM simulations make it seen after $10^{7}$ cycling loading. A crack in the VSFB caused performance issues in the outer band of the bowl where it is clamped to the cross arm members. Therefore deformations must be taking into account before some problems occurred, therefore von Mises stress simulation run and result taking into considerations as displayed in Fig. 5.
Appropriate speed rates calculated to meet the von Mises as displayed in Fig. 6. Maximum speed rate $2736 \mathrm{rpm}$ was found in exited bowl speed to meet the $1352 \mathrm{MPa}$ von Mises criteria of to prevent deformations and large vibrations for cast aluminum.

\section{Results and discussion}

The three leaf-spring legs were used to generate the equation including forces, vibrations and moments for VSFB feeder motion in this investigation. The resultant force and moments caused vibrations in VSFB was analyzed geometrically by leaf springs transformation effect. This produced the balancing force and the momentum of the feeder bowl in VSFB, created the dynamics motion of the bowl feeder, and offered the shifting of the ends of the leaf-spring legs and the angular displacement of the bowl.

\section{Conclusions}

The VSFB designed in this research was clockwise to suit engineering machine elements small parts. The experimental measurement of the displacements was computed by the data using FE method. Experiments and analysis for VSFB design were implemented on a vibration configuration to calculate the reaction to specific axis excitement input data. An electrodynamics vibration system was successfully employed to perform on a single axis inputs. The maximum von Mises stress and position of the specific place on the VSFB was found close to joined axial loads and adjacent uniaxial loads as an important analytical result.

\section{Acknowledgments}

This research is funded by Scientific Research Foundation, project number: 33798, Istanbul University Cerrahpaşa, Turkey.

\section{References}

[1] X. Tian, Z. Yang, Y. Liu, Micromach. 5, 547 (2014).

[2] S.N. Samy, H. ElMaraghy, Int. J. Adv. Manuf. Techn. 62, 813 (2012).

[3] M. Loy, G. Reinhart, Prod. Eng. Res. Devel. 4, 357 (2010).

[4] M.H. Jiang, S.K. Chua, F.L. Tan, Int. J. Prod. Res. 41, 2037 (2003).

[5] G. Reinhart, M. Loy, CIRP J. Manuf. Sci. Techn. 3, 191 (2010).

[6] E. Mucchi, R.D. Gregorio, Mech. Mach. Th. 60, 60 (2013). 